\title{
SPIN-RESOLVED H $\alpha$ SPECTROSCOPY AND PHOTOMETRY OF THE INTERMEDIATE POLAR RXJ0558+5353
}

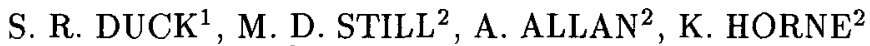 \\ R. W. HILDITCH ${ }^{2}$ \\ 1. Oxford University, Astrophysics, Keble Road, Oxford, UK \\ 2. St. Andrews University, N. Haugh, St. Andrews, Fife, UK
}

\begin{abstract}
RXJ0558+5353 was discovered and classified as an intermediate polar during the ROSAT all-sky survey and subsequent optical follow up programme (Haberl et al. 1994). A further pointed ROSAT observation revealed a spin periodicity of $272.74 \mathrm{~s}$ and a soft X-ray component well represented by an absorbed $57 \mathrm{eV}$ blackbody, the spin modulation being due principally to intensity variations of the component (rather than variable absorption). Optical spectroscopy showed the orbital period to be $4.15 \mathrm{~h}$. In this paper we show the true spin period to be 545.4555(8) s (Allan, Horne $\&$ Hilditch 1995), twice the published X-ray period, and also discuss preliminary results of the first spin-resolved spectroscopy of RXJ0558+5353.
\end{abstract}

\section{Observations}

The photometry presented here, the results of which were first published in Allan, Horne \& Hilditch (1995), uses $V$ and $R$ band light curves obtained at the James Gregory $1 \mathrm{~m}$ telescope, sited at St. Andrews University, between 1994 November 24 and 1995 February 22.

The spin-resolved spectroscopy was obtained in 1995 February on the INT. The set-up used provided a resolution of approximately $80 \mathrm{~km} \mathrm{~s}^{-1}$ and coverage enabling the simultaneous monitoring of $\mathrm{H} \alpha$ and $\mathrm{He} \mathrm{I} 5875$. A total of $173,30 \ldots 45 \mathrm{~s}$, flux calibrated spectra were obtained from about two hours on each of three successive nights in sub-arcsecond seeing.

\section{Results}

A periodogram of the photometric data shows significant power at twice the spin period derived from the ROSAT data. Fig. 1 shows the phase folded light curves from several nights observations, the morphology of which appears to change between the observations. 


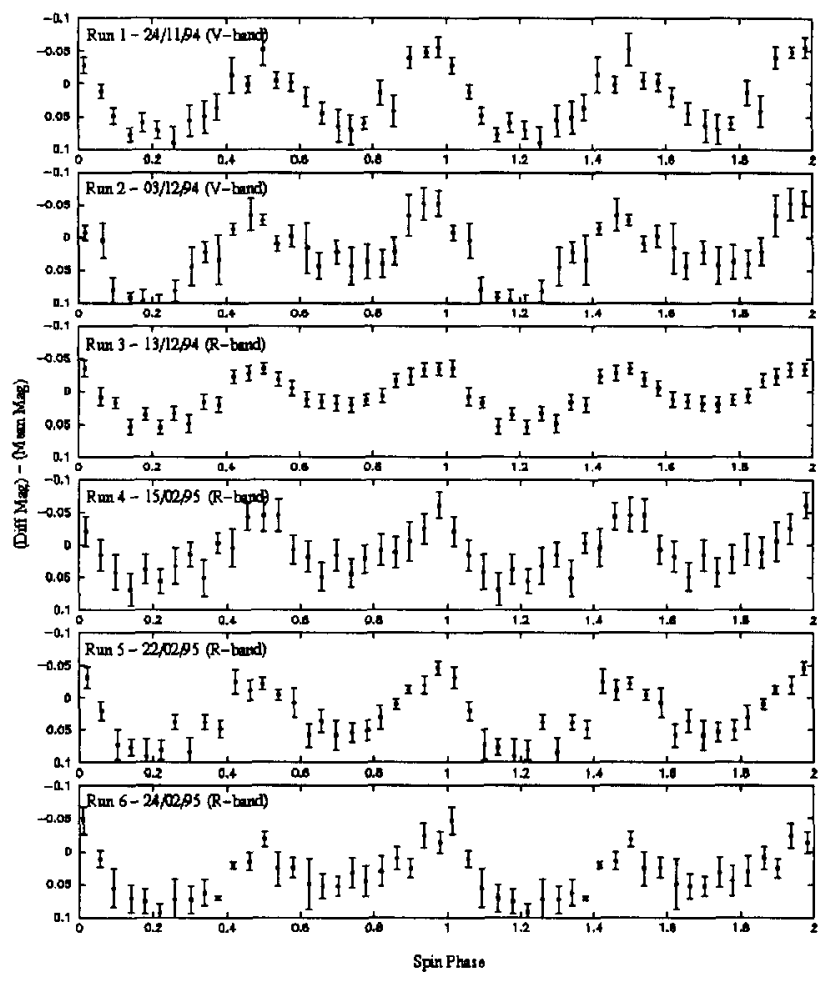

Figure $1 . \quad V$ and $R$ band photometry of RXJ0558+5353 taken on several nights and folded on the new spin period of $\sim 545 \mathrm{~s}$.

The continuum data from the spectroscopic observations also show the same double peaked spin light curve, however the $\mathrm{H} \alpha$ flux spin light curve does not obviously show this, although the orbital scatter may hide a lower level of modulation. Similarly the phase binned $\mathrm{H} \alpha$ line profile shows no clear changes over the spin period.

A Doppler map of the $\mathrm{H} \alpha$ emission presents problems in interpretation, at least partially due to the lack of a published orbital ephemeris. If the bright asymmetrical emission is due to emission from the secondary or hot spot, then although the implied phasing agrees with that of the orbital light curves, the disc-like ring in the tomogram is then not centered anywhere near to the expected location of the primary. This may indicate that this system contains a very distorted disc or that some direct stream accretion is occurring.

\section{References}

Allan, A., Horne, K., Hilditch, R.W., 1995, IAUC6154

Haberl, F., Thorstensen J. R., Schwarzenburg-Czerny, A. et al., 1994, A\&A, 291, 171 